\title{
Abnormalities in Pattern of Lateralization in Relation to Visuospatial Short Term Memory in Children with Williams Syndrome
}

\author{
Antonios Chasouris, ${ }^{1}$ Peter Mayer, ${ }^{2}$ Ian Stuart-Hamilton, ${ }^{2}$ \\ Martin Graff, ${ }^{2}$ and Lance Workman ${ }^{2}$ \\ ${ }^{1}$ Southland Hospital Child Adolescence and Family Service, P.O. Box 828, Invercargill 9840, New Zealand \\ ${ }^{2}$ School of Psychology, Early Years and Therapeutic Studies, Faculty of Life Sciences and Education, \\ Treforest Campus, University of South Wales, Pontypridd CF37 1DL, UK \\ Correspondence should be addressed to Lance Workman; lance.workman@southwales.ac.uk
}

Received 30 September 2013; Revised 20 January 2014; Accepted 3 February 2014; Published 20 March 2014

Academic Editor: Olga Capirci

Copyright @ 2014 Antonios Chasouris et al. This is an open access article distributed under the Creative Commons Attribution License, which permits unrestricted use, distribution, and reproduction in any medium, provided the original work is properly cited.

Williams syndrome (WS) is a genetic disorder characterised by significant intellectual disability. Initial studies indicate that children with WS have a profound bias for information in the top left of visual arrays. Study 1, using a visuospatial memory test for items presented in a $3 \times 3$ matrix, found a significant top left bias in WS children relative to controls. Study 2 used a probe-based memory test with arrays in which items appeared with equal probability in each position. Relative to controls, WS children showed a significant top and left bias. In Study 3, the same children engaged in a visual search task and again, a top and left bias was found in the WS group. It is concluded that children with WS display atypical laterality, which might be explained by abnormal saccadic movements, by abnormalities involving development of the dorsal stream or by uneven cortical development.

\section{Introduction}

Williams syndrome (WS) is a relatively rare genetic disorder that results in mild to severe intellectual disabilities with well-documented peaks and troughs in cognitive and socioemotional abilities (see, e.g., [1]). In particular, it is commonly reported that there are problems with visuospatial orientation, construction, memory, and attention (see, e.g., [1-5]). Children with WS also score very badly on tests of visuospatial memory (e.g., $[5,6]$ ). In addition to these cognitive deficits, there may be indiscriminate, excessive sociability, and problems maintaining satisfactory levels of attention. More than half of children with WS are reported to have difficulty staying on task and being seated during a neurological examination $[7,8]$, compared to matched Down syndrome controls $[9,10]$.

Despite the well-documented evidence for attentional and visuospatial memory problems in WS there is one feature of this disorder that has received relatively little attention in the research literature. Anecdotal reports from clinical practice indicate that children with WS have a strong tendency to concentrate and remember items from the top left corner of a display, and at times it is difficult to distract them from this behaviour. In fact, WS children's teachers often report that they will read the first few words on the line and then move to the next line, irrespective of the fact that the resulting phrase is nonsensical. Whilst such anecdotal reports of a lateral bias have not been formally studied, there has been a great deal of published research demonstrating abnormalities in visuospatial processing for WS individuals. Two particular findings might conceivably be related to this observation. First, it is well established that WS individuals demonstrate poor saccadic control (both horizontal and vertical eye movements requiring more than one correction, [11]) and second according to the "dorsalstream vulnerability" hypothesis such individuals also have deficits in the dorsal occipitoparietal visual stream leading to deficits in motion sensation $[4,5]$. It is entirely possible 
that should this attentional bias towards the top left hand corner stand up to scrutiny, then one or both of these deficits may play a causal role. In addition to these functional abnormalities, it is entirely possible that structural ones might also be involved (see below).

In either event the suggestion of a leftward attentional bias in WS individuals is interesting since it is well established that, at the population level, nonimpaired individuals also demonstrate a small but reliable tendency to attend to visual material that appears to the left of centre (and likewise for an upward bias) [12-16]. This bias has been demonstrated under a number of experimental paradigms including the line bisection task, where individuals tend to "bisect" a line (i.e., decide where the midline is) to the left of centre, and the greyscales task, where participants, who are asked to make a judgment of luminance, tend to select the luminance "ramp" whose relevant feature (bright or dark) is on the left side (see, e.g., $[15-18])$.

This phenomenon has been called "pseudoneglect" since it mirrors the more marked leftward neglect (and strong attentional bias to the right) that is observed in patients suffering from right parietal lobe lesions [13]. Recently it has been reported that this spatial processing pseudoneglect also occurs when short term memory tasks are presented. Della Sala and coworkers have uncovered evidence that, when arbitrary bindings of visual features (i.e., combinations of colour, shape, and location) are presented laterally, participants make fewer errors when recalling bindings made to the left of centre in comparison to the right [19].

It is widely accepted today that this general left lateral bias is a result of structural specialization of the right hemisphere for the deployment of spatial attention [20-23]. In addition to this structural difference between the hemispheres, it has been established that the inherent leftward bias can be exaggerated by activating the right hemisphere (or weakened by activating the left hemisphere [21-23]) Alternatively, Kinsbourne considers that this left lateral bias is largely the result of task-specific activation of the right hemisphere [12]. In either event, given that it is well established that nonimpaired individuals have a tendency to favour the visual stimuli appearing to the left of centre that involves a wide range of different types of stimuli and given that short term memory has recently been shown to be involved in this bias, the possibility that WS individuals demonstrate an extreme form of such a bias is worthy of investigation through tasks engaging short term memory (STM). Such a possibility is congruent with the recent findings of abnormal patterns of structural cerebral asymmetry in WS $[3,6,24,25]$. If WS individuals demonstrate a visual lateral bias then it is possible that this is directly related to these structural asymmetries or to functional abnormalities with regard to saccadic movements and dorsal stream activity as alluded to above. To examine this potential phenomenon of an exaggerated left lateral bias under more controlled conditions, a series of three studies of visuospatial memory was conducted.

\section{Study 1}

2.1. Introduction. A pilot study by the present authors of 20 children with WS aged 9-17 years found that they had a significant bias for recalling items from the left of the array $\left(\chi^{2}=21.96 ; P<0.001\right)$. However, the study, although establishing a strong laterality in children with WS, lacked a control group. Hence, experiment 1 was conducted.

Experiment 1 compared a group of Greek WS children and a group of mental age-matched typically developing children (TD) as the controls. The intent of the study was to determine if the strong left bias was specifically a characteristic of Williams syndrome or occurred in typically developing children also.

2.2. Participants. The WS group was composed of twenty children with a confirmed diagnosis of Williams syndrome (chronological age 6.9-6.4; mean age 10.4, SD 4.10). The children all had a formal diagnosis of WS and all had been genetically tested (cf. [26]). The control group comprised twenty typically developing children (chronological age 5.511.1; mean age 7.3, SD 2.50). The children in the two groups were matched by mental age. The participants' mental age was measured using full Kaufmann Assessment Battery for Children (K-ABC) test; the scores were in the middle quartiles. The full $\mathrm{K}-\mathrm{ABC}$ test gives a composite measure of general intelligence based on both verbal and nonverbal items. It is one of only a very few measures that have been standardised for the Greek population. The typically developing children were recruited from two local schools with the written permission of parents and the head teachers. The study was approved by the faculty ethics committee of the authors' university. Of the children with WS, 14 were male and 6 were female and of the twenty TD children, 10 were male and 10 were female.

2.3. Materials and Procedure. The Kaufman Assessment Battery for Children, or $\mathrm{K}-\mathrm{ABC}$ [27], is a standardized test battery measuring intelligence and achievement in children aged 2.6 to 12.6 years. The K-ABC was developed specifically to assess abilities on tasks that demonstrate a degree of lateralization and is appropriate for use in children with learning difficulties as well as for typically developing individuals [27]. A component of the battery is the visuospatial memory subtest, which consists of a set of pages, each with a display of items [pictures of everyday objects familiar to the participants] located within a $3 \times 3$ matrix. The examiner displays each page in turn to the participant for five seconds. The page is then removed and a blank page consisting of nine blank squares in a $3 \times 3$ array is presented. The participant is asked to point to the squares that contained items in the previously shown display. In the final 11 pages, the number of objects increases and progresses to a $3 \times 4$ display. The test continues until all items are completed.

2.4. Scoring. The number of items correctly recalled was scored and categorised according to the spatial location of the items within the matrix.

Scoring. In addition to the standardised K-ABC scoring, errors were categorised using the following scheme. Horizontal misplacement was defined as misplacing an item in terms 
TABLE 1: Summary of results for Study 1, showing means and standard deviations in parentheses.

\begin{tabular}{lcc}
\hline & $\begin{array}{c}\text { Typically } \\
\text { developing }\end{array}$ & $\begin{array}{c}\text { Williams } \\
\text { syndrome }\end{array}$ \\
\hline $\begin{array}{l}\text { Mean K-ABC score } \\
\text { Horizontal misplacement }\end{array}$ & $11.55(1.57)$ & $4.45(0.89)$ \\
$\quad$ Left & $1.15(1.66)$ & $4.40(3.80)$ \\
Centre & $1.64(1.18)$ & $5.72(2.78)$ \\
$\quad$ Right & $1.50(1.00)$ & $3.70(2.81)$ \\
Vertical misplacement & & \\
Top & $1.05(1.23)$ & $5.05(4.43)$ \\
Centre & $1.74(1.48)$ & $4.55(2.72)$ \\
$\quad$ Bottom & $1.40(0.94)$ & $5.30(3.93)$ \\
Horizontal omission & & \\
Left & $3.60(2.85)$ & $2.60(2.16)$ \\
Centre & $5.05(2.45)$ & $4.84(3.78)$ \\
$\quad$ Right & $2.45(1.57)$ & $8.60(4.89)$ \\
Vertical omission & & \\
Top & $3.00(2.67)$ & $5.50(3.03)$ \\
Centre & $2.44(0.84)$ & $3.65(2.27)$ \\
Bottom & $6.85(3.09)$ & $7.45(3.89)$ \\
\hline
\end{tabular}

of its horizontal position (left middle or centre). For example, an item displayed on the right and remembered being on the left side or the centre was considered to be horizontally misplaced. An analogous procedure was adapted for vertical misplacement (top, middle, and bottom). Horizontal omission was defined as not remembering an item in terms of its displayed position (e.g., an item on the right that was not remembered was said to be omitted from the right). Vertical omission was defined in an analogous fashion (missed top, middle, or bottom items).

2.5. Results. The results are summarised in Table 1. A onetailed $t$-test demonstrated a highly significant superiority of recall for the TD group $\left(t_{(38)}=17.59, P<0.001\right)$.

Horizontal misplacement Analysis was by mixed analysis of variance (ANOVA) using SPSS (which was also used for all subsequent statistical analyses). There was a significant group effect $\left(F_{(1,36)}=22.29 ; P<0.0001\right)$ with WS children misplacing more items on the horizontal axis. There was a significant main effect of position $\left(F_{(2,72)}=26.59 ; P<\right.$ $0.0001)$ and a significant interaction effect $\left(F_{(2,72)}=8.93 ; P<\right.$ $0.0001)$. Within the WS group, $t$-tests with the Bonferroni correction $\left(t_{(19)}=2.29\right.$ in this case) were used as post hoc measures. Misplacement from the left was found to be significantly higher than misplacement to the right $\left(t_{(19)}=\right.$ 5.18; $P<0.05)$, misplacement from the centre was found to be significantly higher than from the left $\left(t_{(19)}=5.42\right.$; $P<0.05)$, and from the centre was found to be significantly higher than from the right $\left(t_{(19)}=5.07 ; P<0.05\right)$. This pattern was not observed in typically developing children matched for mental age.
Horizontal omission Analysis was by mixed ANOVA. WS children omitted significantly more items $\left(F_{(1,38)}=5.99 ; P<\right.$ $0.02)$. There was a significant effect of position $\left(F_{(2,76)}=7.86\right.$; $P<0.001)$ and a significant interaction of group and position $\left(F_{(2,76)}=18.91 ; P<0.0001\right)$. Thus, children with WS omitted significantly more items positioned to the centre or the right than to the left. Within the WS group, post hoc $t$-tests with Bonferroni correction (in this case $t_{(19)}=2.29$ ) indicated that items positioned towards the right on the horizontal axis were omitted significantly more often than items to the left $\left.t_{(19)}=4.94 ; P<0.05\right)$ and items in the centre were omitted more often than items to the left $\left(t_{(19)}=3.48 ; P<0.05\right)$, while the difference between items in the centre and right was nonsignificant $\left(t_{(19)}=1.41\right)$. These findings were not observed in the control group.

Vertical misplacement Analysis was by mixed ANOVA. The WS group misplaced significantly more items on the vertical axis than the control group $\left(F_{(1,38)}=28.17\right.$; $P<0.0001)$. There were no significant effects for position $\left(F_{(2,76)}=0.19 ;\right.$ n.s. $)$ or group-position interaction $\left(F_{(2,76)}=\right.$ 0.87 ; n.s.).

Vertical omission Analysis was by mixed ANOVA. The WS group omitted significantly more items on the vertical axis than the control group $\left(F_{(1,38)}=4.09 ; P<0.05\right)$. A significant main effect of position $\left(F_{(2,76)}=37.29 ; P<0.001\right)$ was found, but no significant interaction. Within the WS group, post hoc $t$-tests with Bonferroni correction (in this case $t_{(19)}=2.29$ ) found significantly greater omission of items at the bottom than the top $\left(t_{(19)}=2.75 ; P<0.05\right)$ and at the bottom than the centre $\left(t_{(19)}=2.99 ; P<0.05\right)$, but no significant difference between centre and top items $\left(t_{(19)}=0.09\right.$; n.s.). No significant effects were found for the TD group.

2.6. Discussion of Study 1. The finding that the TD group has a significantly better visuospatial recall than the WS group supports similar findings in the literature (e.g., $[28,29]$ ). In addition, relative to TD children, there was a leftward bias in children with WS: items positioned to the right were omitted or misplaced significantly more times than other items. How might this be explained? One possibility is that a mild leftward bias found in the general (and unimpaired) population (see $[5,14]$ ) is strongly exaggerated in people with WS. Should this pattern of response stand up to further investigation, however, it is felt that such a leftward bias would be so extreme as to open up the possibility of developmental disruption of the lateralization pattern of processing rather than simply demonstrating an increase the extent of this normal bias.

The evidence of a lateral bias is thus striking, but its cause cannot be readily determined from Study 1 . This is because the K-ABC measure used was a subtest of an IQ test battery; thus, it ostensibly measures memory, not laterality of processing. As such, the frequency with which to-beremembered (TBR) items appear at each position in the display is not totally balanced (i.e., each item does not appear with equal frequency in each position), and the test does not allow for separate measurement of memory and other mental processes. Thus, for example, relatively poor recall of items from one part of a display might be primarily a storage 
problem but could also plausibly be a failure of encoding because items in a particular part of an array are not noticed as often or are more slowly encoded. To disambiguate these issues, it is necessary to conduct two further experiments: one that assesses memory for items in which TBR items appear with equal frequency in all spatial positions and one that assesses ability to process items in different spatial locations when memory load is minimised. To this end, Studies 2 and 3 were conducted.

\section{Study 2}

3.1. Introduction. To address the issues raised above, the following study was designed, in which a TBR item is equally probable at all positions in the display. In contrast to the $\mathrm{K}-\mathrm{ABC}$, which required participants to recall items in multiple locations, the current study aims to test whether participants recognise whether a single item (whose position is varied systematically across the matrix) has appeared in the immediately preceding display. The rationale is that items to the right of the display will be recognised significantly less accurately.

The test materials were presented via a 15 -inch computer screen using the E-Prime software package. The participants were accustomed to using computers.

As with Experiment 1, to allow for the consideration that this might be a universal feature and not one specific to Williams syndrome, a group of mental age-matched typically developing (TD) children was used as controls. The experimental hypotheses were thus that (1) children with Williams syndrome would recall significantly more items from the left of the display and (2) that this bias would be significantly stronger than in typically developing children.

\subsection{Method}

3.2.1. Participants. The participants were the same children who were in Study 1.

3.2.2. Materials. The materials consisted of a set of pictures drawn from copyright-free images specifically designed for use in work with children (examples are given in Figure 1). These were displayed on a 15-inch laptop computer screen in a $3 \times 3$ matrix.

3.2.3. Procedure. All participants were accustomed to using a laptop computer in previous studies. In each trial, nine items from the set of images appeared on the screen for five seconds, with the TBR item having an equal probability of appearing within each position in the matrix. The items were presented quasi-randomly with the proviso that each position was occupied with equal frequency during the course of the trials. This was followed by a test image, and the participant was required by button press to indicate whether they thought it had appeared in the immediately preceding display. The response triggered the presentation of the next display. There was a total of 36 trials, with the TBR item appearing four times in each position.
TABLE 2: Summary of results for Study 2, showing means and standard deviations in parentheses.

\begin{tabular}{lcc}
\hline & \multicolumn{2}{c}{ Group } \\
& Typically developing & Williams syndrome \\
\hline Horizontal accuracy & & \\
Top & $0.98(0.05)$ & $0.94(0.24)$ \\
Middle & $0.91(0.14)$ & $0.79(0.28)$ \\
Bottom & $0.80(0.30)$ & $0.30(0.37)$ \\
Vertical accuracy & & \\
Left & $0.88(0.16)$ & $0.83(0.21)$ \\
Centre & $0.60(0.36)$ & $0.70(0.17)$ \\
Right & $0.65(0.29)$ & $0.20(0.26)$ \\
\hline
\end{tabular}

3.3. Scoring. An accuracy score was recorded for each matrix cell, row, and column. Since each stimulus to be remembered was presented four times in each cell, the accuracy score ranged from 0 (none correctly recognised) to 1 (all correctly recognised) in steps of 0.25 . Thus, the total score for each column and row ranged from 0 to 3.0.

3.4. Results. The findings are summarised in Table 2. The results were subject to a series of statistical analyses.

Horizontal Accuracy Analysis was by mixed ANOVA. There was a significant TD-WS difference $\left(F_{(1,38)}=22.30\right.$; $P<0.001)$, with a significant main effect of position $\left(F_{(2,76)}=\right.$ 26.59; $P<0.001)$ and a significant interaction $\left(F_{(2,76)}=\right.$ 8.93; $P<0.001)$. Namely, WS children were significantly less accurate, and this became more pronounced the lower the TBR item appeared in the display. Within the WS group, post hoc analysis was conducted with $t$-tests with Bonferroni correction (in this case $t_{(19)}=2.94$ ). Centre-top differences were not significant $\left(t_{(19)}=1.66 ; P<0.05\right)$ but centrebottom $\left(t_{(19)}=4.89 ; P<0.05\right)$ and top-bottom $\left(t_{(19)}=4.91\right.$; $P<0.05)$ were. No differences were found in the control group.

Vertical Accuracy Analysis was by mixed ANOVA. A significant group effect was observed $\left(F_{(1,38)}=38.59 ; P<\right.$ $0.001)$ with a significant main effect of position $\left(F_{(2,76)}=\right.$ 48.96; $P<0.001)$ and a significant interaction $\left(F_{(2,76)}=\right.$ 10.94; $P<0.001)$. Within the WS group, post hoc $t$-tests with Bonferroni correction (in this case $t_{(19)}<2.94$ ) found that left was significantly better recalled than centre $\left(t_{(19)}=9.38\right.$; $P<0.05)$, centre better than right $\left(t_{(19)}=3.50 ; P<0.05\right)$, and left better than right $\left(t_{(19)}=6.14 ; P<0.05\right)$. No differences were found in the control group.

3.5. Discussion of Study 2. In summary, Study 2 demonstrates a left wise bias in WS children when asked to recall items. Children with WS find it appreciably harder to recall items the further right they appear in a display. This immediately begs the question of whether the problem is one of retrieval or a failure to process the information appearing in the right of the display. To examine this issue, the following study was devised. 


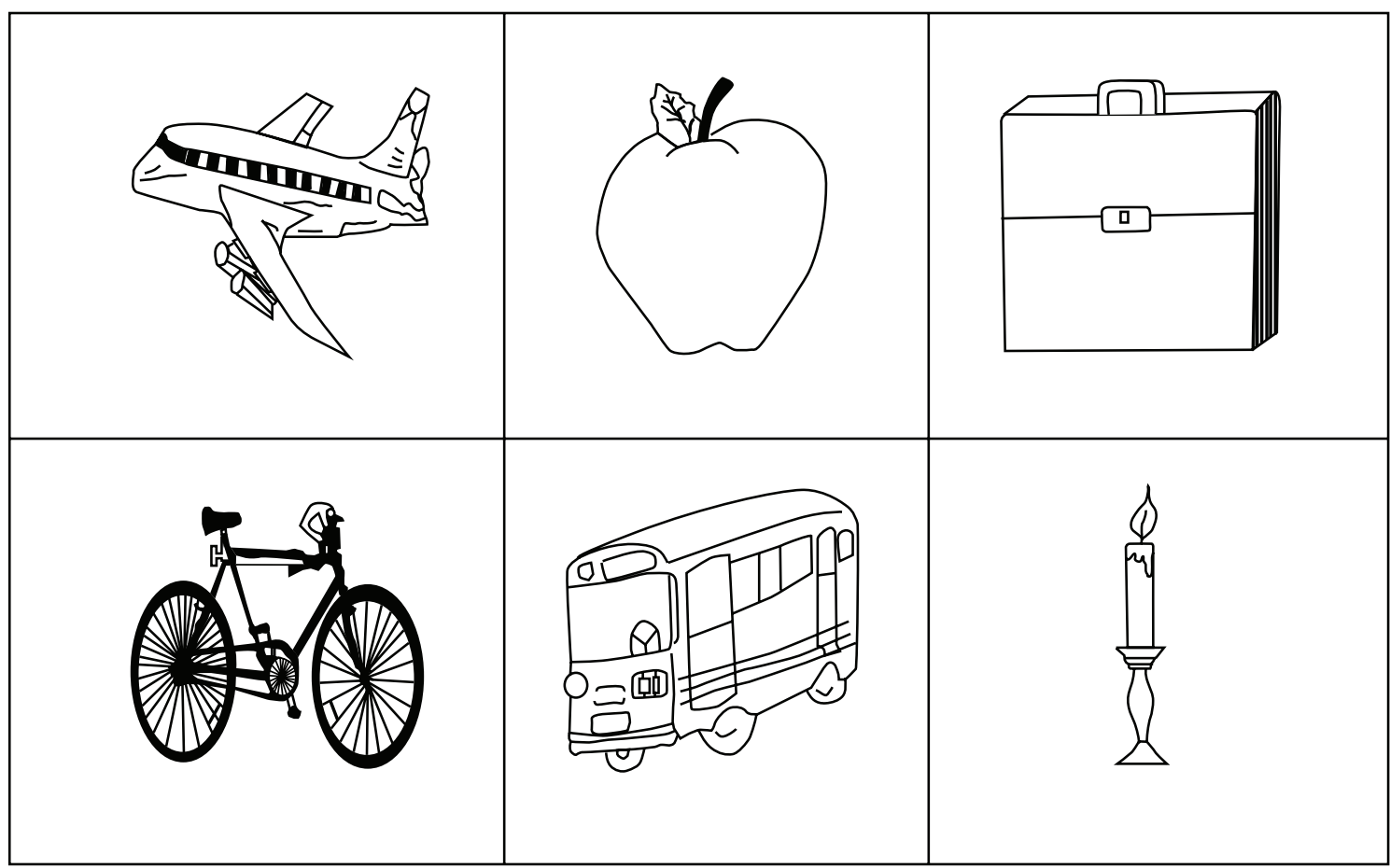

FIgURE 1: Examples of images used in Study 2 [NB not representative of matrix used].

\section{Study 3}

4.1. Introduction. To examine processing/retrieval differences according to position in the array, it is necessary to ask participants to search an array without appreciable memory load to see if items are identified with different latencies and accuracy according to position of the target. If WS participants are able to identify targets equally quickly across all positions in the array, this will indicate a retrieval problem, whereas if there are significantly longer responses in the right of the display, this indicates a problem in processing.

\subsection{Method}

4.2.1. Participants. The participants were identical to those in Studies 1 and 2.

4.2.2. Materials. As in Study 2, using the E-Prime software, a series of arrays was presented on a 15-inch laptop screen. The array consisted of a matrix with 3 rows and 4 columns. Each "cell" contained the same distracter item (an upper case B) with the exception of the target "cell," which contained the target item (an upper case $\mathrm{P}$ ).

4.2.3. Procedure. Participants were required to press one of two response buttons- "Yes" for when they identified the target and "No" if they believed the target was not present. The target was presented at each position in the matrix on 2 occasions, and out of the total number of trials, the target was not present on 4 occasions. There were thus 28 trials in total. Testing terminated when all items had been presented. Prior
TABLE 3: Summary of results for Study 3 (RTs in milliseconds) from target trials only, showing means and standard deviations in parentheses.

\begin{tabular}{lcc}
\hline & \multicolumn{2}{c}{ Group } \\
& Typically developing & Williams syndrome \\
\hline Horizontal latency & & \\
Top & $1308.69(352.22)$ & $4926.73(1012.10)$ \\
Middle & $1335.04(318.53)$ & $7156.23(1826.01)$ \\
Bottom & $1365.89(512.25)$ & $8607.39(2002.95)$ \\
Vertical latency & & \\
Left & $1401.27(316.93)$ & $4753.11(1473.76)$ \\
Middle left & $1399.31(490.70)$ & $7195.84(1780.18)$ \\
$\quad$ Middle right & $1294.52(391.91)$ & $6007.80(1454.92)$ \\
$\quad$ Right & $1369.73(729.75)$ & $8823.52(2617.93)$ \\
\hline
\end{tabular}

to testing, participants were familiarised with the procedure and allowed to practise making responses.

4.3. Scoring. Mean response times (in milliseconds) were recorded and calculated for each position. Responses to each row and column in the display were calculated. Data for analyses of response to position only include those trials where the target was present.

4.4. Results. Results for detection across the rows and columns are presented in Table 3.

Horizontal latencies Analysis was by mixed ANOVA. There was a significant group effect $\left(F_{(1,38)}=301.53 ; P<\right.$ $0.0001)$, with the WS group being slower than controls. There 
was a significant main effect of position on response time $\left(F_{(2,76)}=52.37 ; P<0.001\right)$ and a significant interaction $\left(F_{(2,76)}=49.27 ; P<0.001\right)$. Within the WS group, post hoc $t$ tests with Bonferroni correction (in this case $t=2.94$ ) found a significant top-middle difference $\left(t_{(19)}=6.33 ; P<0.05\right)$, middle-bottom difference $\left(t_{(19)}=4.23 ; P<0.05\right)$, and topbottom difference $\left(t_{(19)}=10.39 ; P<0.05\right)$. No differences were found in the control group.

Vertical latencies Analysis was by mixed ANOVA. There was a significant group effect $\left(F_{(1,38)}=292.07 ; P<0.001\right)$, with the WS group being slower than the controls. There was a significant main effect of position on response time $\left(F_{(3,114)}=23.92 ; P<0.001\right)$ and a significant interaction $\left(F_{(3,114)}=23.86 ; P<0.001\right)$. Within the WS group, post hoc $t$-tests with Bonferroni correction (in this case $t_{(19)}=2.65$ ) found a significant left-middle left $\left(t_{(19)}=4.87 ; P<0.05\right)$, left-middle right $\left(t_{(19)}=4.54 ; P<0.05\right)$, middle left-right $\left.t_{(19)}=3.50 ; P<0.05\right)$, middle right-right $\left(t_{(19)}=5.36\right.$; $P<0.05)$, and left-right $\left(t_{(19)}=6.89 ; P<0.05\right)$ differences in response times, but no significant difference for middle leftmiddle right $\left(t_{(19)}=2.61 ; P<0.05\right)$. No differences were found in the control group.

4.5. Discussion of Study 3. Study 3 demonstrates that the leftward bias observed from clinical observations and in the memory-based Studies 1 and 2 is attributable to a processing bias. The experiment clearly showed that children with Williams syndrome detect target stimuli significantly more slowly as they move towards the right side or the bottom of the matrix. It should be noted that in many cases, the delay in detecting items on the right side of the array is very profound; for example, the mean time to detect an item on the extreme right of the display is almost double that of detecting it on the extreme left. The mean response to target placement at the top and bottom was almost of the same magnitude.

Hence, our sample appears to demonstrate an abnormal pattern of lateralisation. This pattern of response may arguably be related to underlying structural and functional abnormalities (see general discussion below).

Given the extreme times recorded by some WS children to detect the targets when placed on the far right and the finding in Study 2 that pictures situated on the right were not remembered at all or misplaced towards the left, and the possibility of right side neglect must be given consideration. The performance of children with Williams syndrome might be perceived as mirroring the position of patients with a firm diagnoses of neglect, in as much as our WS sample demonstrated an inattention to material presented to the right hemispace, whereas neglect patients suffering from right parietal lobe lesions generally display inattention to material presented to the left hemispace (e.g., $[13,30,31])$. However, the case for a form of reverse neglect is difficult to substantiate, as the participants in this study had no history of brain damage or other brain pathology and had not displayed behaviours that merited being tested for such. Lack of brain imaging findings makes it difficult to determine if unilateral dysfunction might be used to explain our findings. However, subclinical minor lesions, that are undetectable by fMRI, can produce mild to moderate neglect phenomena (e.g., [32, 33]). Further research, augmenting the recent study by Sampaio et al. might be appropriate ([6] see below).

\section{General Discussion}

The findings of the Experiments 1-3 can be summarised as follows.

(1) There is a significant bias towards remembering items presented in the left and left/top area of a spatial array in children with WS both in absolute terms and also relative to typically developing children.

(2) This is may be the result of a processing bias, since children with WS take significantly longer both in absolute terms and relative to typically developing children to detect items in the right and bottom part of the display.

These findings therefore present a phenomenon that has hitherto not been described in detail in the literature, although some studies (e.g., $[34,35]$ ) have noted analogous or potentially supporting findings. Although humans generally have a mild tendency to attend to and recall more from the left of an array, this tendency appears to reach extravagant proportions in children with Williams syndrome, and the phenomenon appears across a range of experimental conditions and is also supported by a variety of clinical observations. How might we explain this finding?

The observation of an apparent left visual field bias might feasibly be related to a number of previous findings with regard to behavioural deficits and abnormal patterns of neurological development in WS individuals. Two particular suggested areas of abnormal development might be pertinent to our observations: atypical saccadic movements and the "dorsal stream vulnerability hypothesis." van der Geest et al., found that, when compared with TD controls, WS individuals demonstrated poor horizontal and vertical saccadic control [11]. Saccades are very rapid goal-directed eyeball movements that ensure a target object is projected onto the fovea. In their study van der Geest et al. found that WS individuals made abnormal "gains" (that is saccades that were either too large or too small, [11]) necessitating more correction saccades to fix a visual target. Since well-controlled saccadic movements are necessary for spatial perception, this finding of a deficit might help to explain the poor scores of our sample on recalling detail of spatial arrays. It does not, however, provide a clear explanation for the lateral bias observed in their scores. Interestingly, when van der Geest et al. analysed the absolute gain difference between leftward and rightward saccades the left-right asymmetry was significantly larger for the WS group than for the control group [11]. It might be premature to suggest that this asymmetry explains our findings, especially given how small and brief saccadic movements are, but given left and rightward saccades are controlled by opposite sides of the brain it is possible that this might contribute to our observations.

In relation to the second hypothesis, there is strong evidence that, in a number of cases of genetically influenced neurological disorder such as Fragile X, autism, and 
Williams Syndrome, whilst the ventral cortical stream (the visual stream that is specialised to identify objects and faces) remains broadly intact, the dorsal cortical (which is important in guiding visual actions) is vulnerable to early developmental disruption $[4,5]$. Such observations have led to the "dorsal stream vulnerability hypothesis"; that is, such genetic abnormalities lead to abnormal development of this cortical stream (which may also be the case following perinatal insult such as hemiplegia). Interestingly, in relation to the current study, in addition to guiding visual actions, the dorsal stream also provides information required for the formation of visuospatial memory and is indirectly involved in attentional control [5]. Hence, another way of explaining the visuospatial short term memory deficits associated with our sample might be by suggesting that this may be related to impairment of the dorsal stream. Once again it may be premature to suggest that the dorsal stream vulnerability hypothesis fully explains our findings, especially given that our study involved a behavioural lateral bias and did not involve activation or lack of activation of specific brain areas. It might however help to provide a tentative clue as to why our WS sample was clearly using a suboptimal search strategy. Perhaps recent neuroimaging findings regarding WS might provide further clues as to underlying neurological abnormalities.

Through use of MRI, Sampaio and her coworkers have uncovered atypical patterns of cerebral asymmetry for the superior temporal gyrus (STG) and likewise for the hippocampal formation in WS individuals $[3,6]$. In the case of the STG, the normal pattern of larger left than right volume was demonstrated to be absent in the WS sample. In contrast the normally observed larger right than left hippocampal formation volume was also absent in the WS individuals. Sampaio and coworkers propose that, in the case of the STG, this abnormal pattern is related to the atypical use of language demonstrated by WS individuals and that the abnormal hippocampal formation is directly related to problems such individuals have with aspects of long term memory formation. The relationship that Sampaio and coworkers propose between abnormal language development and memory deficits and abnormal patterns of lateralized neurodevelopment might conceivably have some parallels with our current findings of abnormal patterns of lateral bias in relation to STM formation.

Moreover, and of particular relevance to the current study, Gaser et al. have uncovered evidence that, although both hemispheres of WS individual show abnormal patterns of gyrification (i.e., degree of convolution of the cortex), the left hemisphere exhibits a greater degree of abnormality than the right [24]. This might be taken as evidence that there is greater developmental disruption in the left hemisphere than the right for WS. If this is the case then it might help to explain the left hemisphere/right visual field neglect in the present study. Hence, the aforementioned structural abnormalities might be directly related to the functional asymmetrical pattern uncovered in our WS sample. In fact, Gaser et al. explicitly predict a structural/functional relationship.

"The observed gyrification abnormalities in individuals with WS might be related to dysfunctions in neuronal circuits and consequently contribute to the distinct cognitive and behavioral profile accompanying the disorder" ([24]).
A further recent observation from the neuroimaging literature might also shed light on the current finding of a lateral preference and open up another possible explanation. Luders and coworkers report that, compared to control participants, WS individuals have a smaller and less curved corpus callosum [36]. Given that the corpus callosum plays an important role both in interhemispheric transfer and in cerebral inhibition [37], it is possible that this abnormality leads, in turn, to a lack of inhibition of the right hemisphere by its left sided partner for certain tasks.

Hence, it is possible that both the poor overall performance and the left visual field bias observed in our WS sample are related to one or more or a number of structural and functional abnormalities including abnormal saccadic movements, dorsal stream disruption, asymmetrical developmental disruption of the hemispheres, or the under development of the corpus callosum. In either event, given the robust nature of the current observation of a left visual field bias, it is not unreasonable to suggest that our findings might be related to such neurological abnormalities. However, whilst such findings with regard to underlying brain mechanisms might conceivably be related to the findings reported here, we must be exercise caution since our findings are based on behavioural observations alone.

Although such explanations are currently speculative, it is of interest to note that there is growing evidence of a relationship between abnormal patterns of lateralization and various other disorders from schizophrenia [38] to Asperger syndrome [39] and autism [40]. In particular, Taylor et al. have uncovered evidence of a lack of a normal pattern of lateralization when responding to emotional chimeric faces (where an emotional half face is presented to one visual field and neutral half face to the other, [40, 41]). In typically developing children, a left visual field advantage for recognising emotions in faces generally develops by the age of 10 [41], whereas in the Taylor et al. study, autistic individuals well beyond this age demonstrated no such lateralized pattern. On the basis of these findings, Taylor et al. suggest that autistic individuals lack a normal pattern of right hemisphere development and that this, in turn, is related to their lack of ability to read emotions in other facial expressions, leading to lack of appropriate social response [40, 41]. In relation to the current study, it is interesting to note that WS individuals are renowned for their prosocial behaviour to others (some researchers have even portrayed them as being the polar opposite of autistic spectrum individuals in their hypersociability (see [42])). Such affectionate responses are, however, frequently indiscriminate and hence they are described as having social-cognitive deficits. It is possible that such overly affectionate behaviour might also be related to this postulated abnormal pattern of structural/functional lateralization and in particular with regard to a lack of inhibition of the right hemisphere. It might be presumptive to suggest that for both WS and autistic spectrum individuals there are problems associated with development of the right hemisphere, but there is at least some evidence of abnormal patterns of lateral response for individuals with both conditions. In order to examine further the relationship between emotional response and patterns of functional lateralization it would be of interest 
to test WS individuals on the chimeric faces task. It would also be of great interest to make use of eye-tracking technology to add further to our knowledge of potential lateral biases in scanning patterns in both WS and autistic spectrum individuals.

In addition, although control groups used in the studies were mental age-matched, it might be informative to compare performance of typically developing children matched for chronological age to see if there are any cognitionindependent maturational factors present. Likewise, it would be useful to identify the performance of children with other forms of intellectual dysfunction on this task (though it is probable that those with highly researched conditions such as Down's syndrome are unlikely to display unusual performance since the sheer bulk of existing research in this field is likely to have identified the phenomenon if it exists). A further consideration is that the size of stimuli and mode of presentation varied between studies. It is unlikely to have affected the lateralisation observed, since stimuli size did not vary very markedly, and lateralisation was found in both paper and video presentations of materials. Nonetheless, a further examination of this factor might be appropriate in future research.

These findings thus arguably raise more questions than answers and it is hoped that future research will lead to appropriate explanations about the observed leftwards bias. In building such explanations researchers would do well to consider each of the functional and structural abnormalities, from dorsal stream disruption to abnormalities of colossal development that have been reported for individuals with Williams syndrome.

\section{Conflict of Interests}

The authors declare that there is no conflict of interests regarding the publication of this paper.

\section{References}

[1] D. Rösch, "Pedagogical guidance in cases of Williams-Beuren syndrome. The role of clinical methods in the long-term guidance of children," Neuropsychiatrie de l'Enfance et de l'Adolescence, vol. 52, no. 6, pp. 430-437, 2004.

[2] J. Burn, "Williams syndrome," Journal of Medical Genetics, vol. 23, no. 5, pp. 389-395, 1986.

[3] A. Sampaio, N. Sousa, M. Férnandez, C. Vasconcelos, M. E. Shenton, and Ó. F. Gonçalves, "MRI assessment of superior temporal gyrus in williams syndrome," Cognitive and Behavioral Neurology, vol. 21, no. 3, pp. 150-156, 2008.

[4] J. Atkinson and O. Braddick, "From genes to brain development to phenotypic behavior. "Dorsal-stream vulnerability" in relation to spatial cognition, attention, and planning of actions in Williams syndrome (WS) and other developmental disorders," Progress in Brain Research, vol. 189, pp. 261-283, 2011.

[5] J. Atkinson, O. Braddick, S. Anker et al., "Neurobiological models of visuospatial cognition in children with Williams syndrome: measures of dorsal-stream and frontal function," Developmental Neuropsychology, vol. 23, no. 1-2, pp. 141-174, 2003.
[6] A. Sampaio, N. Sousa, M. Férnandez, C. Vasconcelos, M. E. Shenton, and Ó. F. Gonçalves, "Williams syndrome and memory: a neuroanatomic and cognitive approach," Journal of Autism and Developmental Disorders, vol. 40, no. 7, pp. 870-877, 2010.

[7] K. Levine, "Information for teachers," Williams Syndrome Association National Newsletter, vol. 11, pp. 3-5, 1993.

[8] J. Vaal, Diversity in Development of Williams Syndrome. Paper presented at the National Family Conference Williams Syndrome Association, San Diego, Calif, USA, 1994.

[9] D. A. Trauner, U. Bellugi, and C. Chase, "Neurologic features of Williams and Down syndromes," Pediatric Neurology, vol. 5, no. 3, pp. 166-168, 1989.

[10] J. H. Brown, M. H. Johnson, S. J. Paterson, R. Gilmore, E. Longhi, and A. Karmiloff-Smith, "Spatial representation and attention in toddlers with Williams syndrome and Down syndrome," Neuropsychologia, vol. 41, no. 8, pp. 1037-1046, 2003.

[11] J. N. van der Geest, G. C. Lagers-Van Haselen, J. M. van Hagen et al., "Saccade dysmetria in Williams-Beuren syndrome," Neuropsychologia, vol. 42, no. 5, pp. 569-576, 2004.

[12] M. Kinsbourne, "The cerebral basis of lateral asymmetries in attention," Acta Psychologica, vol. 33, pp. 193-201, 1970.

[13] D. Bowers and K. M. Heilman, "Pseudoneglect: effects of hemispace on a tactile line bisection task," Neuropsychologia, vol. 18, no. 4-5, pp. 491-498, 1980.

[14] M. E. R. Nicholls, J. B. Mattingley, N. Berberovic, A. Smith, and J. L. Bradshaw, "An investigation of the relationship between free-viewing perceptual asymmetries for vertical and horizontal stimuli," Cognitive Brain Research, vol. 19, no. 3, pp. 289-301, 2004.

[15] G. Jewell and M. E. McCourt, "Pseudoneglect: a review and meta-analysis of performance factors in line bisection tasks," Neuropsychologia, vol. 38, no. 1, pp. 93-110, 2000.

[16] M. E. McCourt, "Performance consistency of normal observers in forced-choice tachistoscopic visual line bisection," Neuropsychologia, vol. 39, no. 10, pp. 1065-1076, 2001.

[17] J. L. Bradshaw, J. A. Bradshaw, and G. Nathan, "Leftwards error in bisecting the gap between two points: Stimulus quality and hand effects," Neuropsychologia, vol. 24, no. 6, pp. 849-855, 1986.

[18] M. E. R. Nicholls, J. L. Bradshaw, and J. B. Mattingley, "Freeviewing perceptual asymmetries for the judgement of brightness, numerosity and size," Neuropsychologia, vol. 37, no. 3, pp. 307-314, 1999.

[19] S. della Sala, S. Darling, and R. H. Logie, "Items on the left are better remembered," Quarterly Journal of Experimental Psychology, vol. 63, no. 5, pp. 848-855, 2010.

[20] J. J. Foxe, M. E. McCourt, and D. C. Javitt, "Right hemisphere control of visuospatial attention: line-bisection judgments evaluated with high-density electrical mapping and source analysis," NeuroImage, vol. 19, no. 3, pp. 710-726, 2003.

[21] M. E. McCourt, P. Freeman, C. Tahmahkera-Stevens, and M. Chaussee, "The influence of unimanual response on pseudoneglect magnitude," Brain and Cognition, vol. 45, no. 1, pp. 52-63, 2001.

[22] M. E. McCourt, M. Garlinghouse, and J. Butler, "The influence of viewing eye on pseudoneglect magnitude," Journal of the International Neuropsychological Society, vol. 7, no. 3, pp. 391395, 2001.

[23] M. E. R. Nicholls and G. R. Roberts, "Can free-viewing perceptual asymmetries be explained by scanning, pre-motor or attentional biases?" Cortex, vol. 38, no. 2, pp. 113-136, 2002. 
[24] C. Gaser, E. Luders, P. M. Thompson et al., "Increased local gyrification mapped in Williams syndrome," NeuroImage, vol. 33, no. 1, pp. 46-54, 2006.

[25] P. M. Thompson, A. D. Lee, R. A. Dutton et al., "Abnormal cortical complexity and thickness profiles mapped in Williams syndrome," Journal of Neuroscience, vol. 25, no. 16, pp. 41464158, 2005.

[26] A. Chasouris, I. Stuart-Hamilton, and P. Mayer, "Variability in developmental milestones and developmental delay in children with Williams syndrome," Educational and Child Psychology, vol. 26, pp. 102-116, 2010.

[27] A. Kaufman and N. Kaufman, The K-ABC Assessment Battery for Children. Circle Pines MN: American Guidance Service, 1983.

[28] P. Wang and U. Bellugi, "Evidence from two genetic syndromes for a dissociation between verbal and visual-spatial short-term memory," Journal of Clinical and Experimental Neuropsychology, vol. 16, no. 2, pp. 317-322, 1994.

[29] E. Semel and S. Rosner, Understanding Williams Syndrome, Lawrence Erlbaum, Hillsdale, NJ, USA, 2003.

[30] K. Bryan, The Right Hemisphere Language Battery, FAR Communications, Boston, Mass, USA, 1995.

[31] R. Desimone and J. Duncan, "Neural mechanisms of selective visual attention," Annual Review of Neuroscience, vol. 18, pp. 193-222, 1995.

[32] T. Namihira, Y. Hirayasu, and Y. Koga, "The assessment of cognitive function in a Williams syndrome patient: a case report," Psychiatry and Clinical Neurosciences, vol. 58, no. 1, pp. 99-100, 2004.

[33] C. Panayiotopoulos, The Epilepsies, Blandon Medical Publishers, London, UK, 2005.

[34] J. J. Crisco, J. M. Dobbs, and R. K. Mulhern, "Cognitive processing of children with Williams syndrome," Developmental Medicine and Child Neurology, vol. 30, no. 5, pp. 650-656, 1988.

[35] M. Nakamura, Y. Kaneoke, K. Watanabe, and R. Kakigi, "Visual information process in Williams syndrome: intact motion detection accompanied by typical visuospatial dysfunctions," European Journal of Neuroscience, vol. 16, no. 9, pp. 1810-1818, 2002.

[36] E. Luders, M. di Paola, F. Tomaiuolo et al., "Callosal morphology in Williams syndrome: a new evaluation of shape and thickness," NeuroReport, vol. 18, no. 3, pp. 203-207, 2007.

[37] J. S. Bloom and G. W. Hynd, "The role of the corpus callosum in interhemispheric transfer of information: excitation or inhibition?" Neuropsychology Review, vol. 15, no. 2, pp. 59-71, 2005.

[38] R. L. C. Mitchell and T. J. Crow, "Right hemisphere language functions and schizophrenia: the forgotten hemisphere?" Brain, vol. 128, no. 5, pp. 963-978, 2005.

[39] C. Ashwin, S. Wheelwright, and S. Baron-Cohen, "Laterality biases to chimeric faces in Asperger syndrome: what is "right" about face-processing?" Journal of Autism and Developmental Disorders, vol. 35, no. 2, pp. 183-196, 2005.

[40] S. Taylor, L. Workman, and H. Yeomans, "Abnormal patterns of cerebral lateralisation as revealed by the universal chimeric faces task in individuals with autistic disorder," Laterality: Asymmetries of Body, Brain and Cognition, vol. 17, no. 4, pp. 428437, 2012.

[41] L. Workman, L. Chilvers, H. Yeomans, and S. Taylor, "Development of cerebral lateralisation for recognition of emotions in chimeric faces in children aged 5 to 11," Laterality: Asymmetries of Body, Brain and Cognition, vol. 11, no. 6, pp. 493-507, 2006.
[42] H. Tager-Flusberg, D. P. Skwerer, and R. M. Joseph, "Model syndromes for investigating social cognitive and affective neuroscience: a comparison of autism and Williams syndrome," Social Cognitive and Affective Neuroscience, vol. 1, no. 3, pp. 175-182, 2006. 

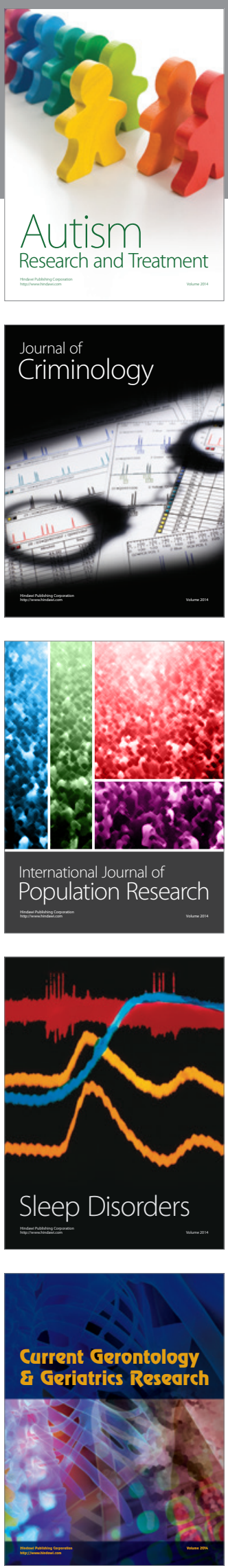
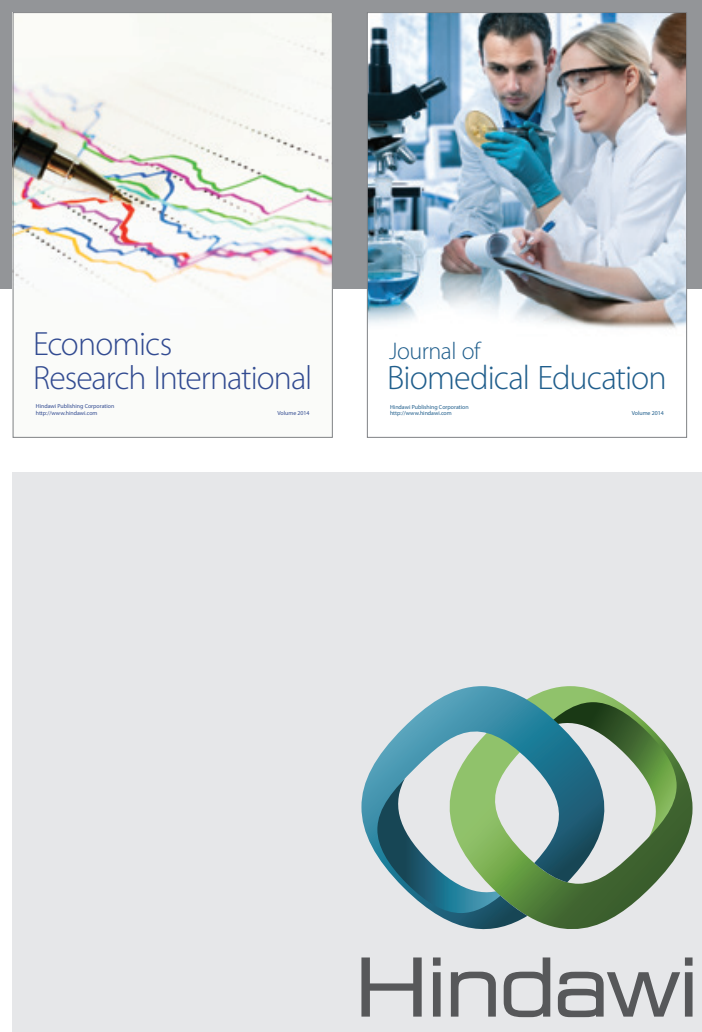

Submit your manuscripts at

http://www.hindawi.com
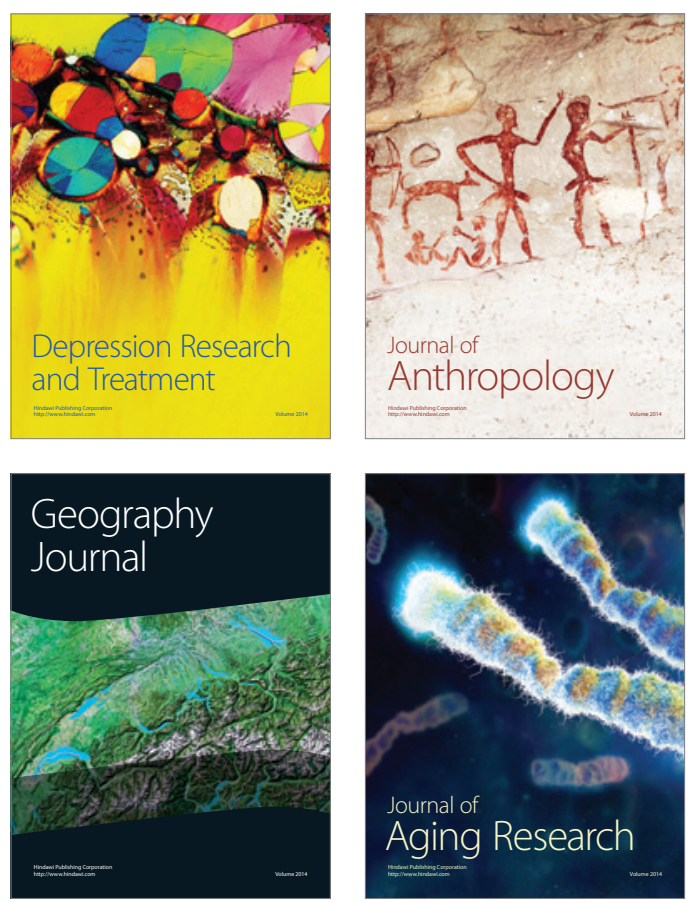
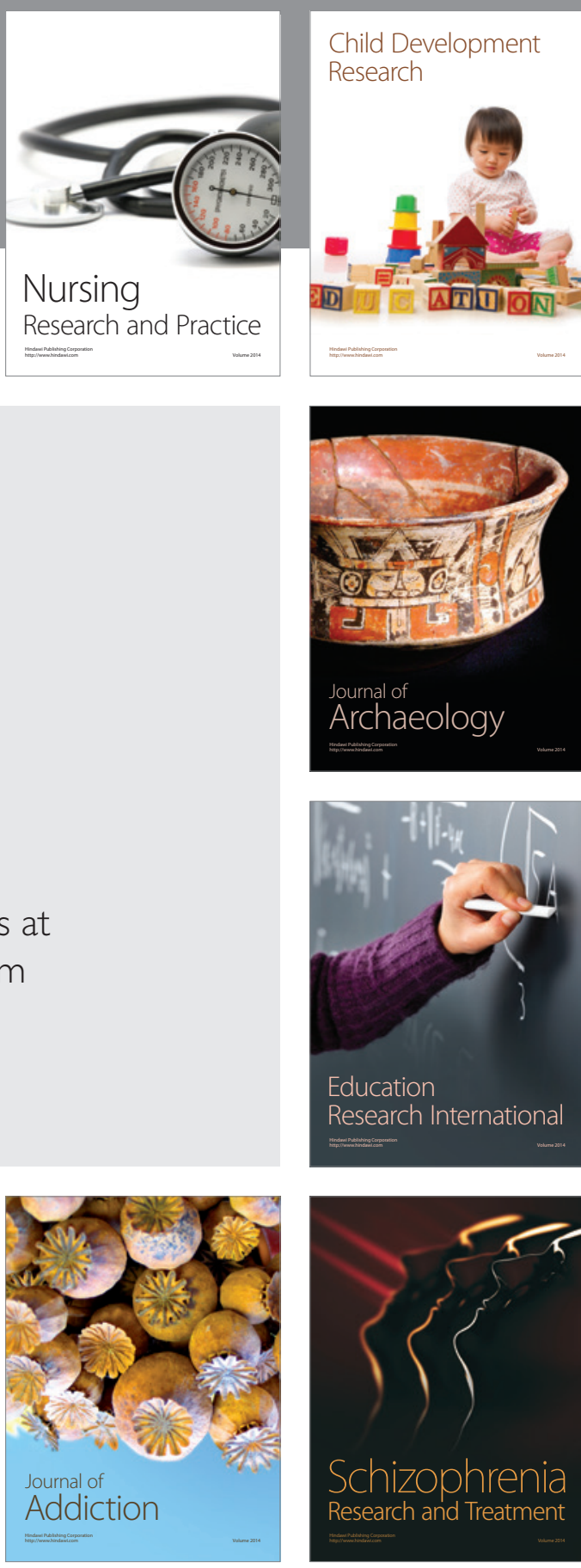

(D)
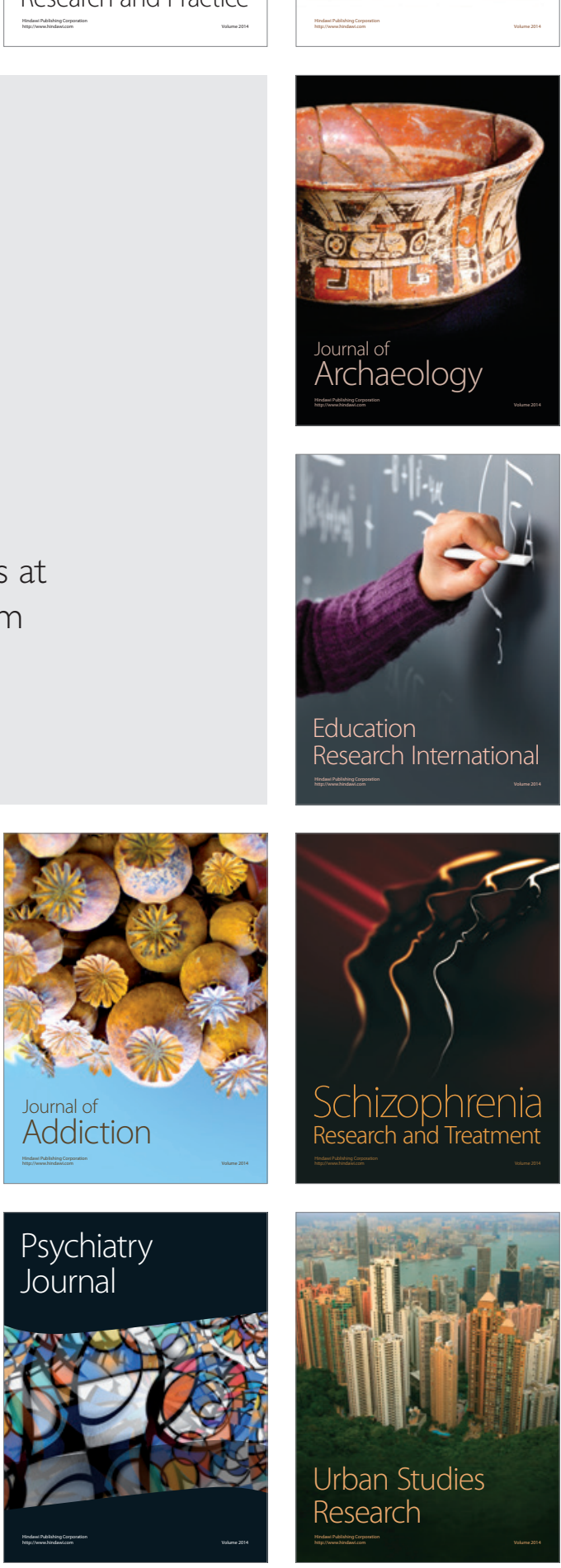